\title{
Nuevos registros de algas verdes marinas (Ulvophyceae) para Tabasco, México
}

\section{New records of marine green algae (Ulvophyceae) from Tabasco, Mexico}

\author{
Nataly Quiroz-González¹,3 (D), Daniel León-Álvarez² (iD y Ma. Guadalupe Rivas-Acuña' (iD
}

1 Universidad Juárez Autónoma de Tabasco, División Académica de Ciencias Biológicas, 0.5 km carretera Cárdenas, 86000 Villahermosa, Tabasco, México.

2 Universidad Nacional Autónoma de México, Facultad de Ciencias, Herbario, Laboratorio de Ficología y Sección de Algas, 04510 CDMX, México.

3 Autor para la correspondencia: natalyquirozgonzalez@gmail.com

Citar como:

Quiroz-González, N., D. León-Álvarez y M. G. Rivas-Acuña. 2017. Nuevos registros de algas verdes marinas (Ulvophyceae) para Tabasco, México. Acta Botanica Mexicana 118: 121-138. DOI: http://dx.doi.org/10.21829/ abm118. 2017.1204

Recibido: 22 de octubre de 2015

Revisado: 9 de mayo de 2016.

Aceptado: 12 de octubre de 2016.

DOl:

http://dx.doi.org/10.21829/abml18. 2017.1204

\section{Resumen:}

Antecedentes y Objetivos: El conocimiento de la ficoflora en el estado de Tabasco es escaso, se tienen 38 registros de Rhodophyta, siete de Ochrophyta, y solo cinco de Chlorophyta. Además, a nivel nacional es el estado costero con el menor número de publicaciones del tema. Se desarrolló el presente trabajo para contribuir al conocimiento de la flora algal del estado, particularmente al de Chlorophyta.

Métodos: Se revisó material procedente de los herbarios de la Facultad de Ciencias de la UNAM (FCME) y de la Universidad Juárez Autónoma de Tabasco (UJAT). Se llevaron a cabo dos periodos de muestreo en temporada de lluvias y secas en siete localidades del litoral tabasqueño. El material recolectado fue preservado en formol a $4 \%$. Se realizaron observaciones de talos completos y cortes con microscopios estereoscópico y óptico. La identificación se llevó a cabo con literatura especializada.

Resultados clave: Se reportan 17 nuevos registros de especies de algas marinas verdes para la costa de Tabasco, pertenecientes a siete géneros, cinco familias y cuatro órdenes. Los órdenes con mayor riqueza específica fueron Ulvales y Cladophorales con ocho y siete especies, respectivamente. Las familias con mayor número de especies fueron Cladophoraceae y Ulvaceae con siete cada una. El género más diverso fue Ulva, con siete especies.

Conclusiones: El número de algas marinas de la clase Ulvophyceae registradas en Tabasco asciende de cinco a 22 reportes, lo que representa $18 \%$ de las especies de Chlorophyta registradas para el Golfo de México. Es la primera vez que se reporta Chaetomorpha nodosa para las costas mexicanas.

Palabras clave: clorofitas, Chaetomorpha nodosa, Golfo de México, macroalgas, riqueza específica.

\section{ABSTRACT:}

Background and Aims: The knowledge of the phycoflora in the state of Tabasco is scarce, with 38 records of Rhodophyta, seven of Ochrophyta, and only five of Chlorophyta. Additionally, at national level it is the coastal state with the lowest number of publications about this topic. Therefore, this study was developed to contribute to the knowledge of its algal flora, particularly of Chlorophyta.

Methods: Material of the herbaria of the Faculty of Sciences of the UNAM (FCME) and the Universidad Juárez Autónoma de Tabasco (UJAT) was revised. Two sampling periods were conducted during the rainy and dry seasons in seven locations on the coast of Tabasco. The collected material was preserved in formaldehyde at $4 \%$. Observations of the thalli and sections were made with stereoscopic and light microscopes. Identification was carried out with specialized literature.

Key results: Seventeen new records of species of green algae are presented for the coast of Tabasco, belonging to seven genera, five families and four orders. The orders with the highest species richness were Cladophorales and Ulvales, with eight and seven species, respectively. Families with the highest species number were Ulvaceae and Cladophoraceae with seven each. The most diverse genus was Ulva, with seven species.

Conclusions: The number of seaweeds of the class Ulvophyceae registered in Tabasco rises from five to 22 records, representing $18 \%$ of Chlorophyta species recorded for the Gulf of Mexico. It is the first time that Chaetomorpha nodosa is reported for the coast of Mexico.

Key words: chlorophytes, Chaetomorpha nodosa, Gulf of Mexico, macroalgae, species richness. 


\section{INTRODUCCIÓN}

La diversidad de algas marinas mexicanas se estima en 1100 especies en el Pacífico, mientras que para el Atlántico oscila en 800 especies (Pedroche et al., 2009). Para el Golfo de México y el Mar Caribe, Ortega et al. (2001) señalan que se han registrado 238 géneros y 651 especies de algas marinas bénticas, siendo dominantes las Rhodophyceae y Chlorophyceae.

Los estudios realizados por diversos autores para conocer la ficoflora del Golfo de México (González-González et al., 1996; Ortega et al., 2001; Garduño-Solórzano et al., 2005) han incluido principalmente los estados de Campeche, Tamaulipas, Veracruz y Yucatán. El conocimiento que se tiene de la flora ficológica de Tabasco es escaso, siendo notablemente excluido en las exploraciones ficológicas, lo que se refleja en el bajo número de especies registradas para el estado. A nivel nacional, Tabasco ocupa el último lugar en diversidad de algas marinas (Dreckmann et al., 2006) en relación a todos los grupos, incluyendo las Chlorophyta, de las cuales existen 132 especies reportadas para el Golfo de México (Pedroche y Sentíes, 2003).

Los estudios de mayor importancia realizados sobre las algas marinas en Tabasco son los de Orozco y Dreckmann (1995), Ramírez (1995), Dreckmann y De Lara-Issasi (2000) y Sentíes y Dreckmann (2013), donde en total se dan a conocer 50 taxa de macroalgas marinas para el litoral tabasqueño, de las cuales cinco corresponden a la clase Ulvophyceae.

El objetivo de este trabajo es contribuir al conocimiento de la ficoflora del estado de Tabasco, particularmente en lo que respecta a las Ulvophyceae. Se presenta un listado florístico, así como las descripciones e ilustraciones de los nuevos registros para el área de estudio.

\section{Materiales y MÉtodos}

\section{Zona de estudio}

El estado de Tabasco está ubicado en el sureste de la República Mexicana entre las coordenadas $17^{\circ} 15^{\prime} 00^{\prime \prime}$ y $18^{\circ} 39^{\prime} 07^{\prime \prime}$ de latitud norte y $90^{\circ} 50^{\prime} 23^{\prime \prime}$ y $94^{\circ} 07^{\prime} 49^{\prime \prime}$ de lon- gitud oeste. Limita al norte con el Golfo de México, al noroeste con Campeche, al sureste con Guatemala, al sur con Chiapas y al oeste con Veracruz. El clima es de tipo cálido-húmedo, con una media anual de $26^{\circ} \mathrm{C}$ (West et al., 1985).

$\mathrm{Su}$ zona costera posee una ligera inclinación hacia el Golfo de México, se caracteriza por ser una planicie sedimentaria originada de la fuente interior de los ríos y la consecuente depositación se hace a través de humedales, estuarios, llanuras de inundación, manglares y/o pantanos marinos. Las playas bajas arenosas están constituidas principalmente por arcillas, limos, arenas y materia orgánica; también se presentan sedimentos no consolidados, los cuales están sujetos a la acción constante del oleaje (West et al., 1985). Dichas características no favorecen el desarrollo de muchas especies de algas bentónicas.

El litoral del estado de Tabasco presenta $191 \mathrm{~km}$ de longitud (Sánchez y Barba, 2005). Las localidades de estudio pertenecen a los municipios de Cárdenas, Centla y Paraíso (Cuadro 1 y Figura 1).

Cárdenas presenta cerca de $67 \mathrm{~km}$ de costa y posee varias lagunas costeras de extensión considerable, tales como la laguna El Carmen, laguna El Pajonal, laguna Machona y laguna Redonda. No se encuentran desembocaduras de ríos en este municipio. Centla tiene cerca de $75 \mathrm{~km}$ de costa en donde desembocan los ríos González, Grijalva, y San Pedro y San Pablo. Paraíso posee alrededor de $38 \mathrm{~km}$ de costa y en su territorio se encuentra la laguna de Mecoacán, la cual es de gran importancia económica. En su costa desembocan los ríos Seco, González y Verde.

\section{Trabajo de herbario y de campo}

Se realizó una revisión del material ficológico procedente de Tabasco en las colecciones de los herbarios de la Facultad de Ciencias de la Universidad Nacional Autónoma de México (FCME) y en el herbario de la Universidad Juárez Autónoma de Tabasco (UJAT). Con los datos recabados se determinaron los sitios y la temporada de muestreo para el trabajo de campo. 
Cuadro 1: Localidades de muestreo en el estado de Tabasco, México.

\begin{tabular}{llc}
\hline Municipio & Localidad & Georreferencia \\
\hline Cárdenas & Puerto Andrés Sánchez Magallanes & $18^{\circ} 17^{\prime} 42.91^{\prime \prime} \mathrm{N} / 93^{\circ} 50^{\prime} 49.33^{\prime \prime O}$ \\
& Playa las Brisas & $18^{\circ} 17^{\prime} 26.65^{\prime \prime} \mathrm{N} / 93^{\circ} 52^{\prime} 20.38^{\prime \prime O}$ \\
Centla & Playa Pico de Oro & $18^{\circ} 27^{\prime} 30.86^{\prime \prime} \mathrm{N} / 92^{\circ} 51^{\prime} 17.57^{\prime \prime O}$ \\
& Playa Miramar & $18^{\circ} 29^{\prime} 00.34^{\prime \prime} \mathrm{N} / 92^{\circ} 47^{\prime} 44.65^{\prime \prime O}$ \\
Paraíso & Playa Paraíso & $18^{\circ} 25^{\prime} 47.93^{\prime \prime} \mathrm{N} / 93^{\circ} 12^{\prime} 4.60^{\prime \prime O}$ \\
& Puerto de Dos Bocas & $18^{\circ} 26^{\prime} 39.77^{\prime \prime} \mathrm{N} / 93^{\circ} 07^{\prime} 12.29{ }^{\prime \prime O}$ \\
& Poblado Chiltepec & $18^{\circ} 25^{\prime} 43.29^{\prime \prime} \mathrm{N} / 93^{\circ} 05^{\prime} 00.88^{\prime \prime O}$ \\
\hline
\end{tabular}

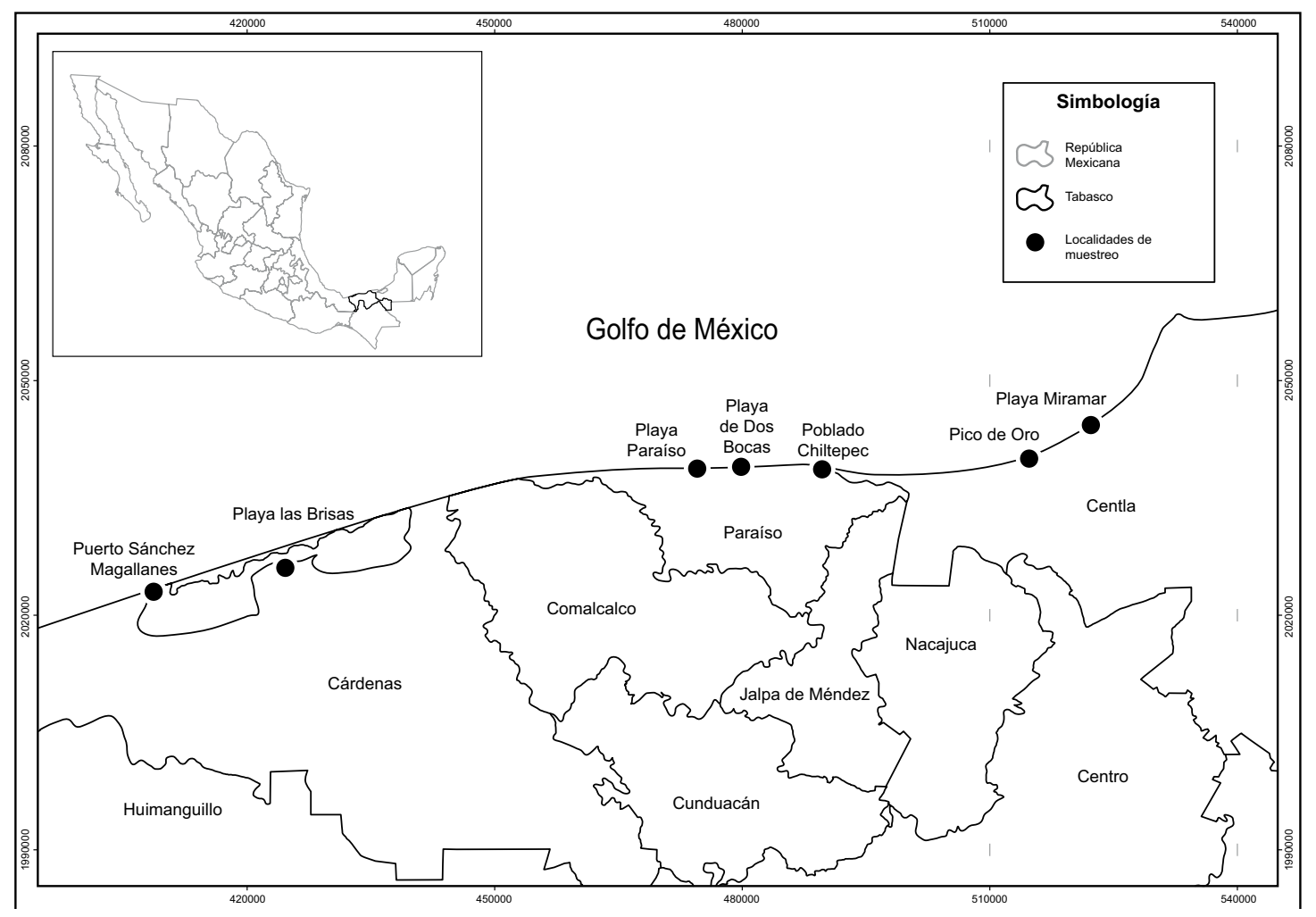

Figura 1: Ubicación del área de estudio y de las localidades de muestreo.

Paralelamente se efectuaron tres exploraciones ficológicas en la zona de estudio durante la época de secas (en mayo de 2012) y la época de lluvias (en octubre de 2012). Los organismos se recolectaron manualmente con espátula y navaja de campo en la zona intermareal y en las escolleras. Para cada ejemplar se tomaron las georre- ferencias con un GPS (GARMIN 60 CSx, Kansas, EUA), se anotaron datos ecológicos como el epifitismo y tipo de sustrato, datos ambientales como exposición al oleaje y nivel de marea, así como biológicos, forma de crecimiento (marañas, mechones, cespitosos o matorrales), coloración, textura (liso, áspero o aterciopelado) y consistencia 
(algodonoso, blando, carnoso, coriáceo, esponjoso, pétreo o semiduro). Las muestras se trasladaron al herbario de la Universidad Juárez Autónoma de Tabasco (UJAT) y se fijaron con formol a $4 \%$ en agua de mar (Lot y Chiang, 1986).

\section{Procesamiento y descripción de muestras}

Los ejemplares se observaron con estereoscopio (Olympus SZ2-IL-ST, Tokio, Japón y ZEIGEN ZEZEZ2100, Ciudad de México, México). Se realizaron preparaciones semipermanentes y en fresco, se llevaron a cabo cortes histológicos o de secciones del talo en forma transversal. Los cortes se realizaron manualmente con una navaja de disección, los montajes semipermanentes se fijaron con gelatina glicerinada a $75 \%$, teñida con cristal violeta (Castellaro et al., 2007). Para la observación y toma de medidas celulares se empleó un microscopio compuesto (Olympus modelo CX31RTSF, Tokio, Japón). Se realizaron las descripciones e ilustraciones de los ejemplares.

\section{Identificación taxonómica}

Se determinó el material ficológico con el uso de las siguientes obras: Taylor (1960), Littler et al. (1989), Littler y Littler (2000) y Peruzzi et al. (2009). Para la actualización nomenclatural y sistemática se utilizó AlgaeBase (Guiry y Guiry, 2016). Además, se compararon caracteres taxonómicos con los citados por Mendoza-González y Mateo-Cid (1996), Ceballos (2008), Ardito y García (2009), Miranda et al. (2009), Morón y Ardito (2010), y Solé y Pardo (2010).

Se integraron los números de cada preparación y las especies identificadas en la base de datos del sistema de información de la sección de algas del herbario de la Universidad Nacional Autónoma de México y del herbario de la Universidad Juárez Autónoma de Tabasco. Las muestras fueron depositadas en el herbario UJAT.

\section{Resultados}

De acuerdo con la clasificación actual establecida por Guiry y Guiry (2016), las algas verdes de este estudio se incluyen en la clase Ulvophyceae. Se identificaron 17 es- pecies de Ulvophyceae marinas pertenecientes a siete géneros, cinco familias y cuatro órdenes (Cuadro 2), se presenta además un ejemplar que fue solo identificado a nivel de género. Todas las especies listadas en este trabajo son nuevos reportes para Tabasco. Además, se presentan tres nuevos registros de orden, cuatro de familia y seis nuevos registros a nivel de género. Chaetomorpha nodosa Kütz. es reportada por primera vez para las costas mexicanas.

Los órdenes que presentaron mayor riqueza de especies fueron Ulvales y Cladophorales con ocho y siete especies respectivamente. A su vez las familias con mayor número de especies fueron Cladophoraceae y Ulvaceae con siete cada una, mientras que el género más diverso fue Ulva L. con siete especies que representan más de la tercera parte de los nuevos registros.

El número de taxa de Ulvophyceae para Tabasco ascendió de cinco a 22, lo que representa $18 \%$ de las especies de Chlorophyta reportadas para el Golfo de México. La ficoflora del estado aumentó de 50 a 67 taxa, incluyendo los reportes previos para este grupo, así como los de algas rojas y pardas. El número de localidades exploradas en Tabasco se incrementó a 12 sitios, al sumar los lugares previamente estudiados por Ramírez (1995), Orozco y Dreckmann (1995), Sentíes y Dreckmann (2013) y los considerados en este estudio (Cuadro 3). La localidad con mayor número de especies fue el Puerto Andrés Sánchez Magallanes (Cárdenas) con 10 especies; sin embargo, el municipio con mayor riqueza de especies fue Paraíso, con 12. Por otro lado, el municipio de Centla presentó el menor número (1) (Cuadro 3).

Las algas se encontraron sobre distintos sustratos: $47.4 \%$ de las especies sobre arena y sustratos artificiales como escolleras, basura, costales y telas, $26.3 \%$ en sustratos como otras algas, troncos y conchas de animales, mientras que $15.8 \%$ fueron exclusivamente flotadoras, el resto (10.5\%) se encontraron flotando y en un sustrato biótico. Las escolleras fueron el sustrato más frecuente.

Los sustratos de tipo biótico más frecuentes fueron algas y conchas de ostión (Crassostrea virginica Gmelin). Rhizoclonium riparium (Roth) Harvey se encontró como epífito de Gracilaria sp. También se colectaron ejem- 
Cuadro 2: Nuevos registros de Ulvophyceae marinas de Tabasco, usando la clasificación de Guiry y Guiry (2016). *Género previamente reportado por Sentíes y Dreckmann (2013). En negritas el nuevo registro para las costas mexicanas.

\begin{tabular}{|c|c|c|c|c|}
\hline Clase & Orden & Familia & Género & Especie \\
\hline \multirow[t]{18}{*}{ Ulvophyceae } & Bryopsidales & Caulerpaceae & *Caulerpa & $\begin{array}{l}\text { C. sertularioides f. brevipes (J. } \\
\text { Agardh) Sved. }\end{array}$ \\
\hline & \multirow[t]{8}{*}{ Cladophorales } & \multirow[t]{8}{*}{ Cladophoraceae } & \multirow[t]{3}{*}{ Chaetomorpha } & C. antennina (Bory) Kütz. \\
\hline & & & & C. gracilis Kütz. \\
\hline & & & & C. nodosa Kütz. \\
\hline & & & \multirow[t]{2}{*}{ Cladophora } & Cladophora sp. \\
\hline & & & & C. vagabunda (L.) Hoek \\
\hline & & & \multirow[t]{3}{*}{ Rhizoclonium } & R. crassipellitum W.y G.S. West \\
\hline & & & & R. riparium (Roth) Harv. \\
\hline & & & & R. tortuosum (Dillwyn) Kütz. \\
\hline & Ulotrichales & Ulotrichaceae & Ulothrix & U. flacca (Dillwyn) Thur. \\
\hline & \multirow[t]{8}{*}{ Ulvales } & Kornmanniaceae & Blidingia & $\begin{array}{l}\text { B. marginata P.J.L. Dang. ex } \\
\text { Bliding }\end{array}$ \\
\hline & & \multirow[t]{7}{*}{ Ulvaceae } & \multirow[t]{7}{*}{ Ulva } & U. compressa $\mathrm{L}$. \\
\hline & & & & U. fasciata Delile \\
\hline & & & & U. flexuosa Wulfen \\
\hline & & & & U. intestinalis $\mathrm{L}$. \\
\hline & & & & U. lactuca $\mathrm{L}$. \\
\hline & & & & U. prolifera O.F. Müller \\
\hline & & & & U. rigida C. Agardh \\
\hline
\end{tabular}

plares que fueron hospederos de otras especies: Chaetomorpha antennina (Bory) Kütz. fue hospedero para Sahlingia subintegra (Rosenv.) Kornmann y Erythrotrichia carnea (Dillwyn) J. Agardh, Chaetomorpha nodosa para E. carnea, y Ulva rigida C. Agardh para Grateloupia filicina (Lamouroux) C. Agardh.

Respecto a las temporadas, se observaron nueve especies solo en la temporada de lluvias, seis únicamente para la temporada de secas y tres para ambas estaciones (Cuadro 3).

\section{Descripciones morfológicas}

Blidingia marginata (J. Agardh) P.J.L. Dang. ex Bliding.

Figs. 3G-H.
Talo filamentoso, color verde pálido; filamentos libres, enredados, como una borla, unidos al sustrato por un disco basal, de $5 \mathrm{~cm}$ de largo, de 55-75 $\mu \mathrm{m}$ de ancho; ramificaciones ligeramente curvadas; células dispuestas en hileras longitudinales particularmente a lo largo de los márgenes, cuadradas a poliédricas de 7-13 $\mu \mathrm{m}$ de diámetro; cloroplasto estrellado con un único pirenoide central prominente.

Ejemplares examinados: MÉXICO. Tabasco, Cárdenas, Playa las Brisas, 25.X.2009, H. Martínez CA103 (UJAT). Paraíso: Playa Paraíso Escollera Oeste, 25.X.2012, N. Quiroz et al. CA126 (UJAT).

Referencias: Taylor, 1960; Peruzzi et al., 2009. 
Cuadro 3: Especies registradas por localidad de estudio y su relación con el sustrato. X: presencia de la especie por localidad. Localidades. SM: Puerto Andrés Sánchez Magallanes; PB: Playa las Brisas; PO: Pico de Oro; PM: Playa Miramar; PDB: Puerto de Dos Bocas; PPEO: Playa Paraíso Escollera Oeste; PCH: Poblado Chiltepec. Sustrato. Ro: rocas (Escolleras); An: animales; Fl: flotadoras; Ar: arena, Al: algas; Co: costales; Tr: troncos; Te: telas. Acrónimos. CA: Colección Algas (UJAT); GM: Golfo de México (FCME). L: recolectada en temporada de lluvias; S: recolectada en temporada de secas.

\begin{tabular}{|c|c|c|c|c|c|c|c|c|c|}
\hline \multirow{3}{*}{$\begin{array}{l}\text { Referencias de } \\
\text { herbario }\end{array}$} & \multirow[t]{3}{*}{ Taxa } & \multicolumn{7}{|c|}{ Localidades } & \multirow[t]{3}{*}{ Sustrato } \\
\hline & & \multicolumn{2}{|c|}{ Cárdenas } & \multicolumn{2}{|c|}{ Centla } & \multicolumn{3}{|c|}{ Paraíso } & \\
\hline & & SM & PB & $\mathrm{PO}$ & PM & PDB & PPEO & $\mathrm{PCH}$ & \\
\hline CA2 & Caulerpa sertularioides f. brevipes & & & & & & $x-L$ & & Ro \\
\hline $\begin{array}{l}\text { GM79, GM391, } \\
\text { GM399,GM402, } \\
\text { GM406, CA115, } \\
\text { CA132, CA163, } \\
\text { CA204 }\end{array}$ & Chaetomorpha antennina & $\mathrm{x}-\mathrm{L}, \mathrm{S}$ & & & & & $x-L$ & & An, Ro \\
\hline CA129 & C.gracilis & $x-L$ & & & & & & & $\mathrm{Fl}$ \\
\hline CA246 & C. nodosa & $x-L$ & & & & & & & $\mathrm{An}, \mathrm{Fl}$ \\
\hline CA244,CA132 & Cladophora sp. & $\mathrm{x}-\mathrm{S}$ & & & & & & & $\mathrm{Ar}$ \\
\hline $\begin{array}{l}\text { GM391, GM400, } \\
\text { GM401, GM402 }\end{array}$ & C. vagabunda & & & & & & $x-L$ & & Ro \\
\hline CA121 & Rhizoclonium crassipellitum & & & & $x-S$ & & & & $\mathrm{Fl}$ \\
\hline CA84 & Rhizoclonium riparium & & & & & $x-S$ & & & $\mathrm{Al}, \mathrm{Fl}$ \\
\hline CA88 & Rhizoclonium tortuosum & & & & & $x-S$ & & & $\mathrm{Fl}$ \\
\hline CA246 & Ulothrix flacca & $x-L$ & & & & & & & Co \\
\hline CA103, CA126 & Blidingia marginata & & $x-L$ & & & & $x-S$ & & Ro \\
\hline CA112 & Ulva compressa & & & & & & & $x-S$ & $\mathrm{Ar}$ \\
\hline $\begin{array}{r}\text { GM391, GM395, } \\
\text { GM401, CA132 }\end{array}$ & U. fasciata & $x-L$ & & & & & $x-L$ & & An, Ro \\
\hline $\begin{array}{l}\text { GM71, GM401, } \\
\text { GM406, CA2 }\end{array}$ & U. flexиosa & $x-L$ & & & & & $x-L$ & & Ro \\
\hline GM391,CA114, C246 & U. intestinalis & $x-L$ & & & & & $x-L$ & $x-L$ & An, $\operatorname{Tr}, \operatorname{Ro}, \mathrm{Co}$ \\
\hline CA89, CA211 & U. lactuca & $x-L$ & & & & $\mathrm{x}-\mathrm{S}$ & & & An,Ro \\
\hline CA2 & U. prolifera & & & & & $x-S$ & $\mathrm{x}-\mathrm{S}$ & & Ro \\
\hline $\begin{array}{l}\text { CA131, CA132, } \\
\text { CA163, CA203, } \\
\text { CA243 }\end{array}$ & U. rigida & $x-L$ & & & & & & & An, Ro, Co, Te \\
\hline
\end{tabular}

Caulerpa sertularioides f. brevipes (J. Agardh) Sved. Figs. 2A-D.

Talo color verde claro, con un estolón principal de 2-2.5 $\mathrm{mm}$ de diámetro, de $10 \mathrm{~cm}$ de largo a partir del cual surgen frondas erectas y rizoides postrados; frondas erectas de 1.2-2.5 cm de largo, de 0.4-0.9 cm de ancho, ramificación de primer orden, pinnadas, aplanadas, rámulas opuestas, curvadas hacia arriba, estrechas en la base, ápice con mucrón, 180-330 $\mu \mathrm{m}$ de diámetro, de 3-11 mm de largo.

Ejemplares examinados: MÉXICO. Tabasco, Paraíso, Playa Paraíso Escollera Oeste, 15.VIII.1989, D. León CA2 (UJAT).

Referencia: Taylor, 1960. 


\section{Chaetomorpha antennina (Bory) Kütz. Figs. 2E-G.}

Talo filamentoso, color verde brillante, no ramificado, gregario, con forma de mechón, unidos por un disco ba$\mathrm{sal}$; filamentos de 1.4-5.5 cm de longitud; células cilíndricas de 530-700 $\mu \mathrm{m}$ de diámetro, 2-3 diámetros de longitud; célula basal cónica de 100-700 $\mu \mathrm{m}$ de diámetro, de 10.4-17 diámetros de longitud, varias constricciones anulares cercanas a la base; los rizoides se ramifican y poseen leves estrías longitudinales; célula apical redondeada.

Ejemplares examinados: MÉXICO. Tabasco, Paraíso, Playa Paraíso Escollera Oeste, 15.VIII.1989, D. León GM79, GM391, GM399, GM402, GM406 (FCME). Cárdenas, Puerto Andrés Sánchez Magallanes, 02.IV.2011, J. Zamudio CA115 (UJAT); 27.X.2012, N. Quiroz et al. CA132 (UJAT); 27.X.2012, I. Torres y C. Guerrero CA163 (UJAT); 27.X.2012, J. Arias y I. Torres CA204 (UJAT).

Referencia: Littler y Littler, 2000.

Chaetomorpha gracilis Kütz. Figs. 2H-I.

Talo filamentoso, color verde brillante; filamentos uniseriados libres y enredados, no ramificados, ligeramente curvados, con pared celular gruesa; células cilíndricas de 60-75 $\mu \mathrm{m}$ de diámetro, de 2 diámetros de longitud; célula basal de 125-250 $\mu \mathrm{m}$ de largo, con abundantes cloroplastos reticulados.

Ejemplares examinados: MÉXICO. Tabasco, Cárdenas, Puerto Andrés Sánchez Magallanes, 27.X.2012, N. Quiroz et al. CA129 (UJAT).

Referencias: Taylor, 1960; Ardito y García, 2009.

\section{Chaetomorpha nodosa Kütz. Fig. 3A.}

Talo filamentoso, color verde oscuro, no ramificado, aislado o formando grupos de dos a tres individuos; filamentos de 1-4 cm de largo, unidos al sustrato por una célula basal recta con base discoide; célula basal cónica, más larga que ancha, de 20-25 $\mu$ m diámetro, de 2.5-6 diámetros de longitud; el resto de las células con paredes lameladas y leves constricciones, de 31-41 $\mu \mathrm{m}$ de diámetro, de 0.6-1 diámetros de longitud; célula apical redondeada.

Ejemplares examinados: MÉXICO. Tabasco, Cárdenas, Puerto Andrés Sánchez Magallanes, 02.IX.2012, O. Hernández CA246 (UJAT).

Referencia: Taylor, 1960; Dawes y Mathieson, 2008 .

Nota: Nuevo registro para las costas de México de acuerdo con Guiry y Guiry (2016).

Cladophora vagabunda (L.) Hoek. Fig. 3C.

Talo filamentoso, color verde-pardo a verde brillante, gregario, con un eje principal, ramificaciones pseudodicotómicas y pseudotricotómicas hacia la base, hacia la parte superior ramificación unilateral en distintos planos, ramificaciones de hasta sexto orden; zona de crecimiento hacia la base; células de los ejes principales de 168-280 $\mu \mathrm{m}$ de diámetro, de 10-22 diámetros de longitud; células del resto del filamento de 120-200 $\mu \mathrm{m}$ de diámetro, de 7 diámetros de longitud; células apicales cónicas, con parte distal redonda, obtusa de 30-40 $\mu \mathrm{m}$ de diámetro, de 5-7 diámetros de longitud.

Ejemplares examinados: MÉXICO. Tabasco, Paraíso, Playa Paraíso Escollera Oeste, 15.VIII.1989, D. León GM391, GM400, GM401, GM402 (FCME).

Referencias: Ceballos, 2008; Dawes y Mathieson, 2008 .

Cladophora sp. Fig. 3B.

Talo filamentoso, color verde brillante, con un eje principal, ramificación alterna en más de un plano hasta de tercer orden, en la parte superior la ramificación es unilateral; paredes celulares gruesas, abundantes cloroplastos; células de los ejes principales de 120-200 $\mu \mathrm{m}$, de 7 diámetros de longitud; células del resto del filamento de 

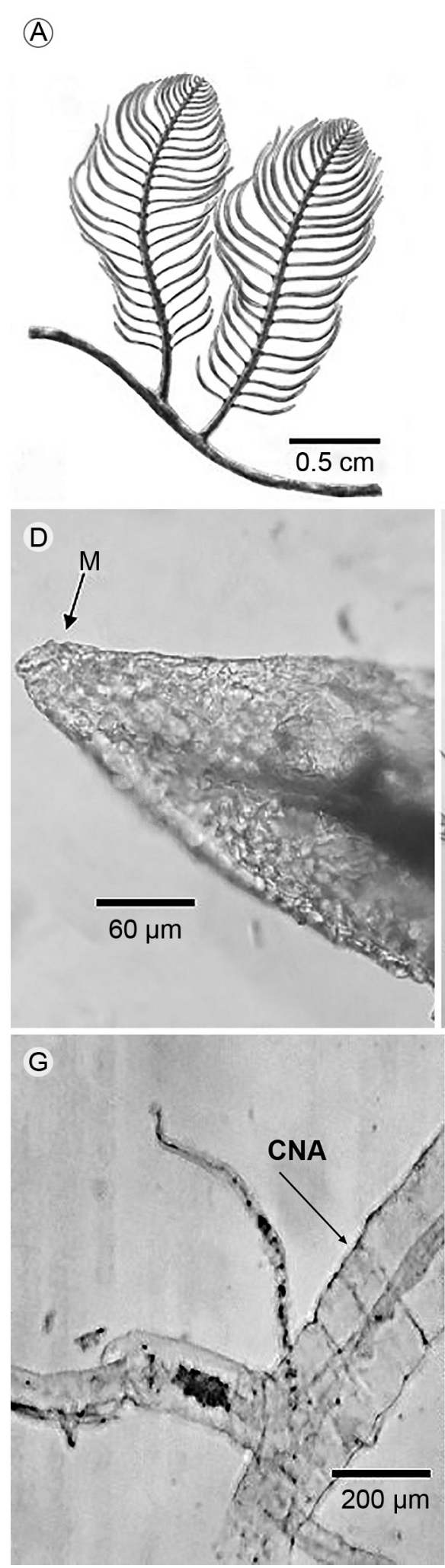
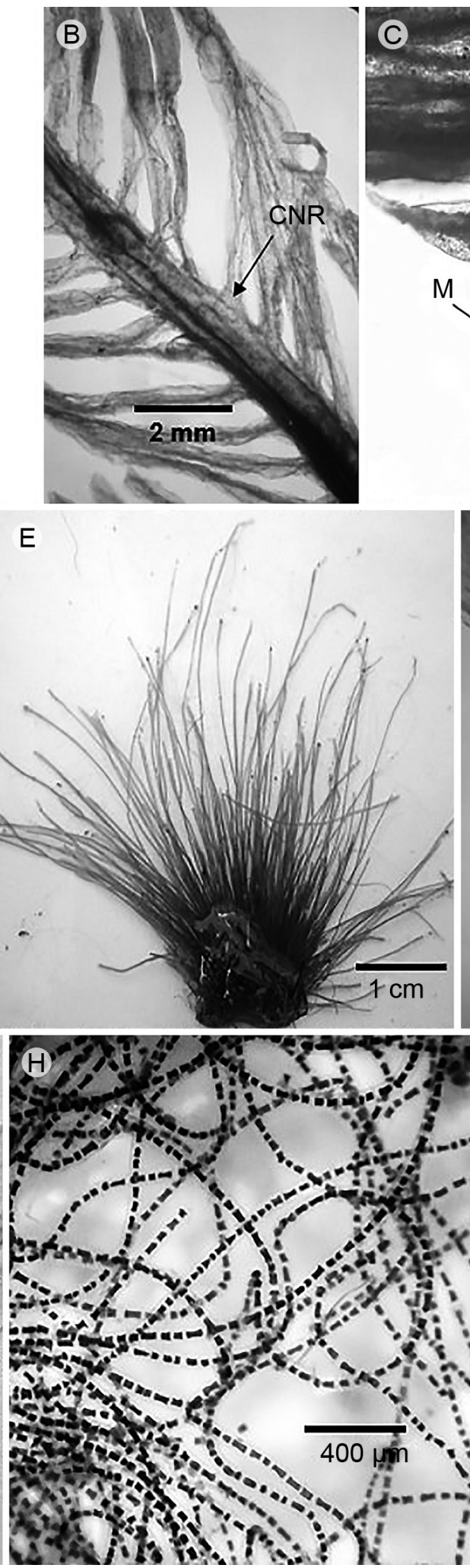
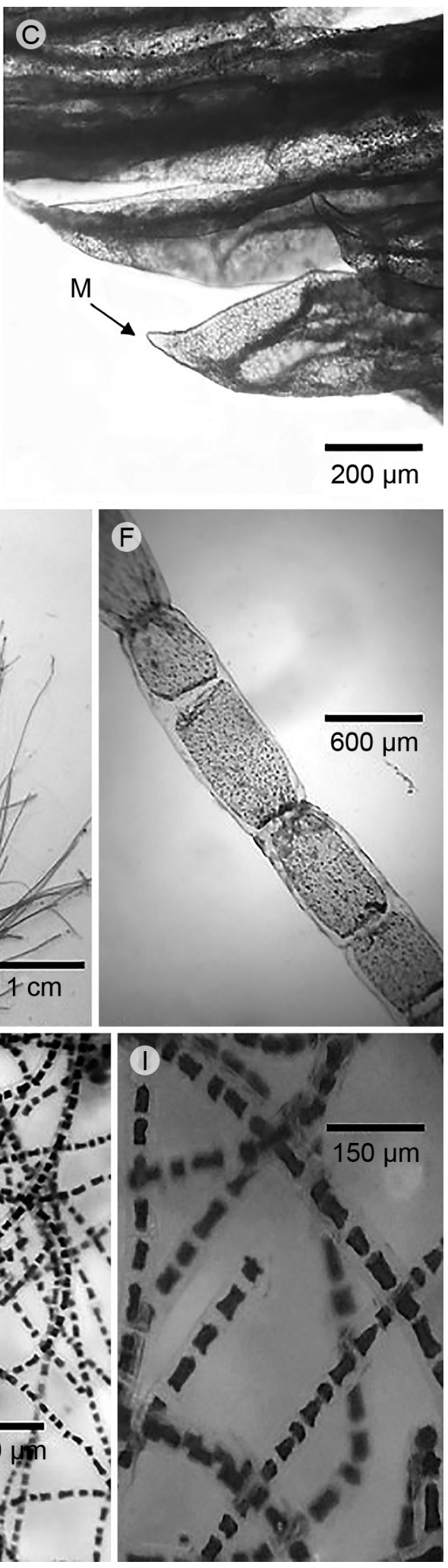

Figura 2: A. talo de Caulerpa sertularioides f. brevipes (J. Agardh) Sved.; B. detalle de ramulas de C. sertularioides f. brevipes, constricción basal (CNR); C. y D. detalle del ápice de las rámulas (M, mucrón); E. talo de Chaetomorpha antennina (Bory) Kütz.; F. detalle del filamento en C. antennina; G. célula basal y rizoides de C. antennina, constricciones anulares (CNA); H. talo de Chaetomorpha gracilis Kütz.; I. detalle del talo de $C$. gracilis. 
102-113 $\mu \mathrm{m}$ de diámetro, de 0.5-2 diámetros de longitud; células apicales con forma cónica, con punta redonda de 34-90 $\mu \mathrm{m}$ de diámetro, de 3 diámetros de longitud.

Ejemplares examinados: MÉXICO. Tabasco, Cárdenas, Puerto Andrés Sánchez Magallanes, 02.IX.2012, O. Hernández CA244 (UJAT); 27.X.2012, N. Quiroz et al. CA132 (UJAT).

Referencia: Taylor, 1960.

Rhizoclonium crassipellitum W. \& G.S. West. Fig. 3D.

Talo filamentoso, color verde limón, enredado, con consistencia algodonosa; filamentos ligeramente curvados, sin ramificaciones, de 30-43 $\mu \mathrm{m}$ de diámetro; células de 48-115 $\mu \mathrm{m}$ de largo, equivalente a 2-3 diámetros de longitud; cloroplastos adosados a las paredes y reticulados.

Ejemplares examinados: MÉXICO. Tabasco, Centla, Playa Miramar, 27.X.2012, N. Quiroz, et al. CA121 (UJAT).

Referencia: Taylor, 1960.

\section{Rhizoclonium riparium (Roth) Harv. Fig. 3E.}

Talo filamentoso, color verde amarillento, con consistencia algodonosa; filamentos curvados, con escasas ramificaciones, de 10-17 $\mu \mathrm{m}$ de diámetro; células de 38-84 $\mu \mathrm{m}$ de largo, equivalente a 4-6 diámetros de longitud; cloroplastos adosados en las paredes parietalmente.

Ejemplares examinados: MÉXICO. Tabasco, Paraíso, Puerto de Dos Bocas, 12.IV.2008, S. Marín CA84 (UJAT).

Referencia: Taylor, 1960.

Rhizoclonium tortuosum (Dillwyn) Kütz. Fig. 3F.

Talo filamentoso color verde oscuro, formando masas flotantes, crecimiento parecido a una borla; predominantemente erectos, sin una estructura visible de fijación, consistencia algodonosa; células de 50-70 $\mu \mathrm{m}$ de diámetro, de 48-115 $\mu \mathrm{m}$ de largo, equivalente a 1-2 diámetros de largo; paredes celulares gruesas de 17-20 $\mu \mathrm{m}$ de ancho; cloroplastos reticulados.

Ejemplares examinados: MÉXICO. Tabasco, Paraíso, Puerto de Dos Bocas, 26.V.2008, K. González CA88 (UJAT).

Referencia: Taylor, 1960.

Ulothrix flacca (Dillwyn) Thur. Fig. 3I.

Talo filamentoso, color verde brillante; filamentos uniseriados sin ramificaciones con múltiples septos, de 23-39 $\mu \mathrm{m}$ de diámetro; células de 10-37 $\mu \mathrm{m}$ de diámetro con paredes anchas, de 6-7 $\mu \mathrm{m}$ de ancho, de 28-45 $\mu \mathrm{m}$ de largo; cloroplastos en forma de anillo y con disposición parietal en las células; de 1 a 2 pirenoides.

Ejemplares examinados: MÉXICO. Tabasco, Cárdenas, Puerto Andrés Sánchez Magallanes, 02.X.2012, O. Hernández CA246 (UJAT).

Referencia: Taylor, 1960.

Ulva compressa L. Figs. 4A, G.

Talo monostromático, tubular y hueco, color verde-amarillento, comprimido hacia el ápice; bordes lisos, con constricciones, talos torcidos, ramifican hacia la base y sus ramas parten de un mismo eje; talo de 0.1-0.4 cm de ancho, de 1.3-3.6 cm de largo; células en vista superficial no muestran un patrón de organización definida, forma irregular o rectangular con bordes angulares, de 6-14 $\mu \mathrm{m}$ de diámetro.

Ejemplares examinados: MÉXICO. Tabasco, Paraíso, Playa el Varadero, 25.X.2012, D. González CA112 (UJAT).

Referencias: Taylor, 1960; Hayden et al., 2003; Ceballos, 2008; Dawes y Mathieson, 2008.

Ulva fasciata Delile. Figs. 4B, H; 5D.

Talo biestromático con forma de cintas, color verde manzana, unidos por una base en forma de cuña; márgenes li- 
(A)

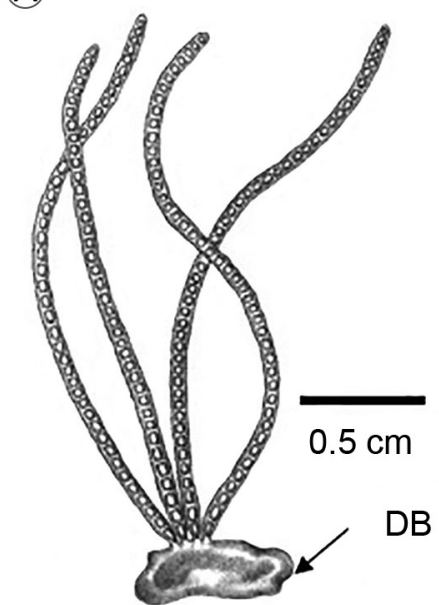

(D)
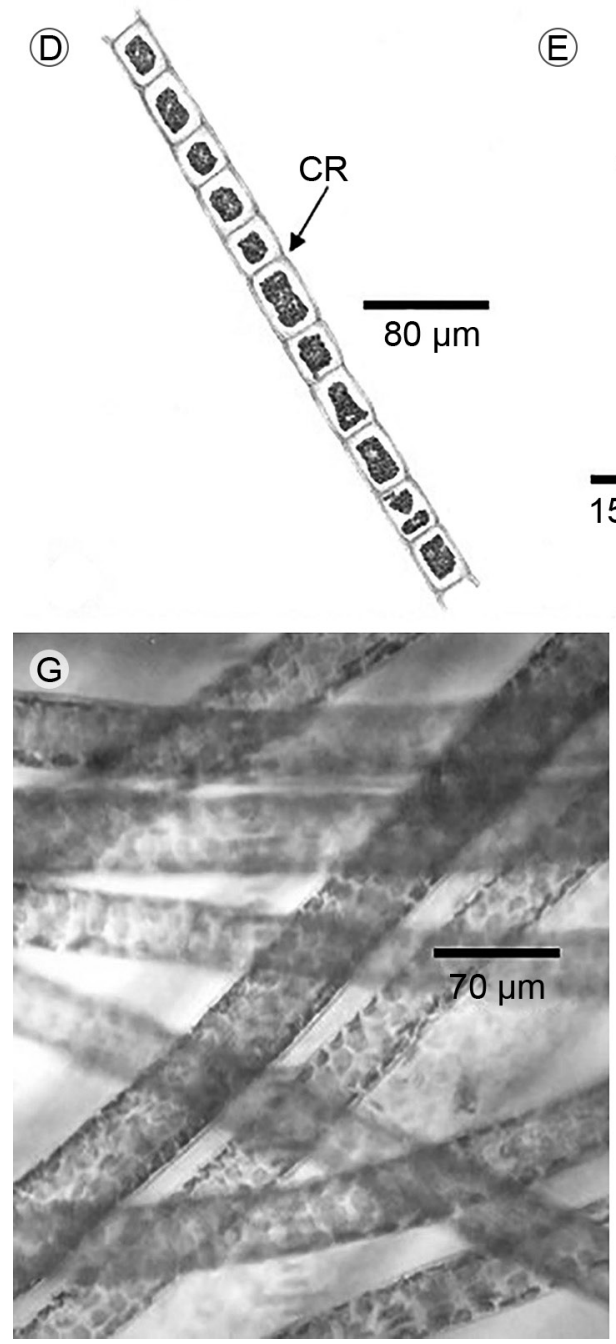

(B)

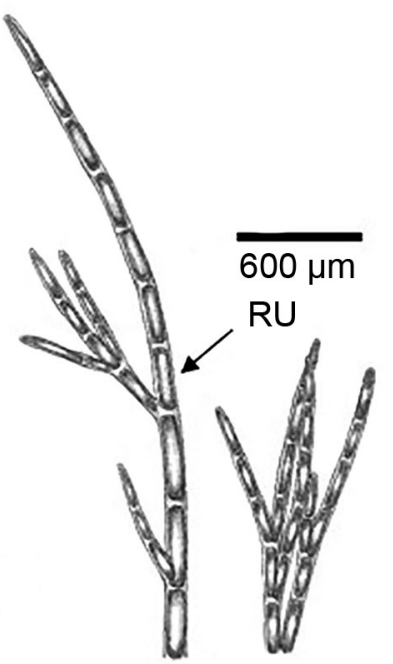

(E)

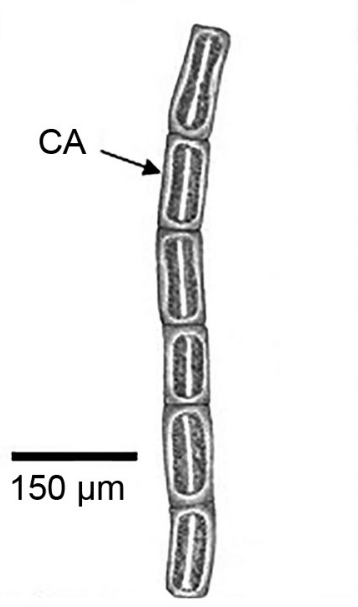

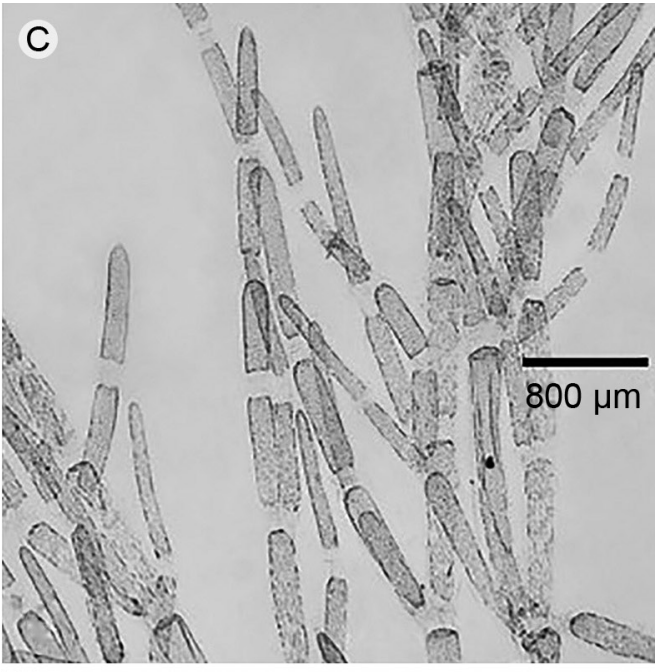
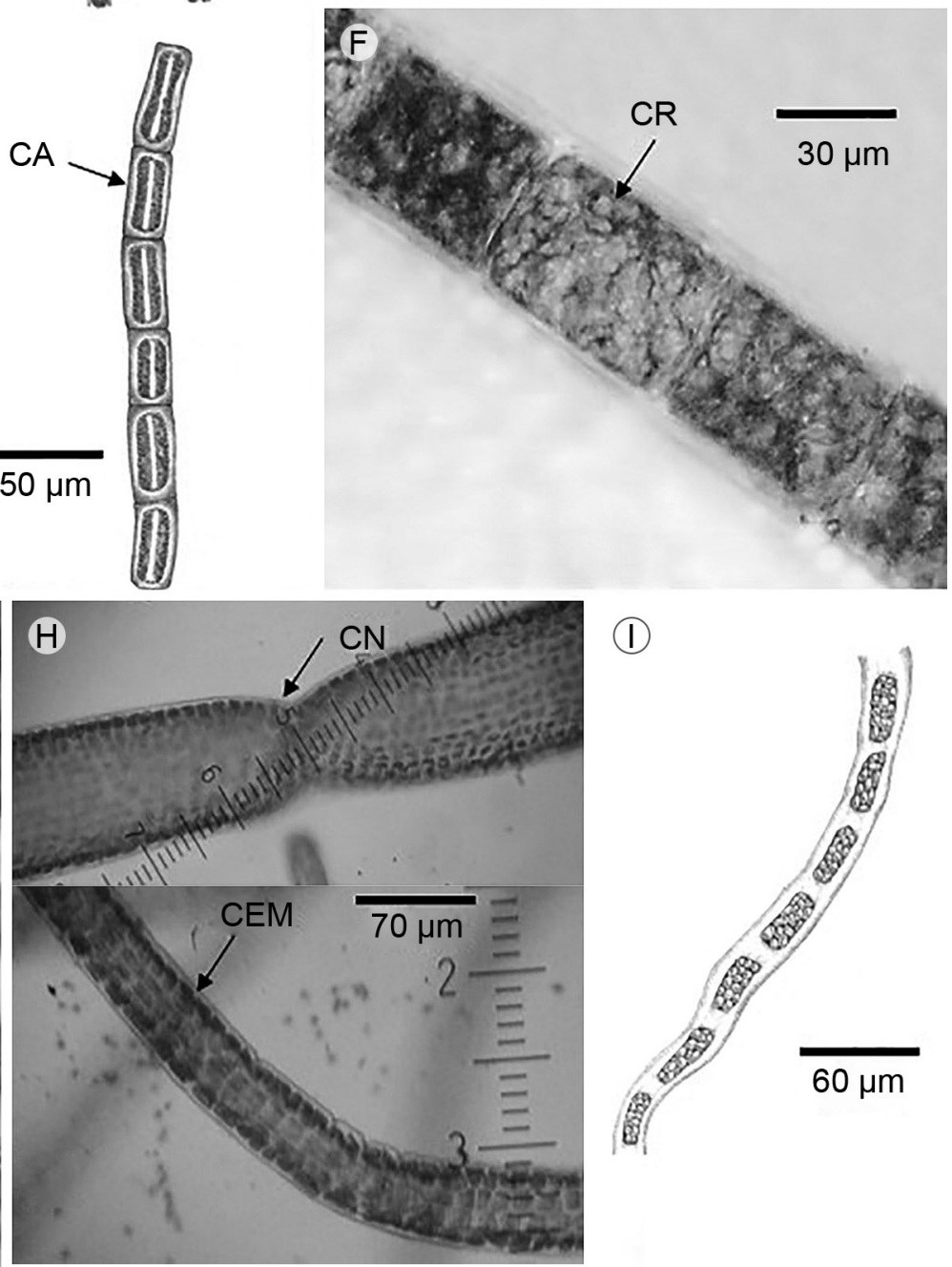

(1)

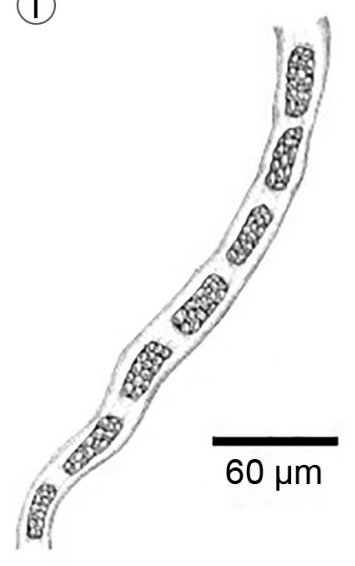

Figura 3: A. talo de Chaetomorpha nodosa Kütz., disco basal (DB); B. talo de Cladophora sp., parte media y apical, hacia el ápice la ramificación es unilateral (RU); C. Cladophora vagabunda (L.) Hoek.; D. Rhizoclonium crassipellitum W. \& G.S. West., con cloroplastos reticulados (CR); E. Rhizoclonium riparium (Roth) Harv., cloroplastos adosados a las paredes (CA); F. Rhizoclonium tortuosum (Dillwyn) Kütz., cloroplastos reticulados (CR); G. Talo de Blidingia marginata (J. Agardh) P.J.L. Dang. ex Bliding.; H. detalle del talo de B. marginata, constricciones en el talo (CN) y las células marginales poliédricas (CEM); I. talo de Ulothrix flacca (Dillwyn) Thur. 
geramente lobados u ondulados; talo de tamaño irregular de 1-1.8 cm de ancho, de 6.2-9.4 cm de largo; en vista superficial las células presentan disposición irregular, ovaladas a irregulares de 12-16 $\mu \mathrm{m}$ de ancho, de 15-17.5 $\mu \mathrm{m}$ de largo, más alargadas hacia los márgenes y hacia la base.

Ejemplares examinados: MÉXICO. Tabasco, Paraíso, Playa Paraíso Escollera Oeste, 15.VIII.1989, D. León GM391, GM395, GM401 (FCME), CA2 (UJAT). Cárdenas, Puerto Andrés Sánchez Magallanes, 27.X.2012, N. Quiroz et al. CA132 (UJAT).

Referencias: Taylor, 1960; Littler y Littler, 2000; Hayden et al., 2003; Ceballos, 2008; Dawes y Mathieson, 2008.

\section{Ulva flexuosa Wulfen. Fig. 5F.}

Talo monostromático, tubular y hueco, color verde brillante, sin ramificaciones, gregarios, creciendo como un mechón; filamentos de 1.7-1.2 cm de longitud, de 0.5-2 $\mathrm{mm}$ de diámetro; margen liso con algunas constricciones; células organizadas en filas de formas diversas, rectangulares, ovaladas o cuneadas de $16 \mu \mathrm{m}$ de largo, de $11 \mu \mathrm{m}$ de diámetro.

Ejemplares examinados: MÉXICO. Tabasco, Paraíso, Playa Paraíso Escollera Oeste, 15.VIII.1989, D. León GM71, GM401, GM406 (FCME), CA2 (UJAT); Cárdenas, Puerto Andrés Sánchez Magallanes, 27.X.2012, N. Quiroz et al. CA132 (UJAT).

Referencias: Mendoza-González y Mateo-Cid, 1996; Littler y Littler, 2000; Hayden et al., 2003; Ceba1los, 2008; Dawes y Mathieson, 2008.

\section{Ulva intestinalis L. Figs. 4C; 5A, E.}

Talos tubulares huecos, gregarios, de color verde-amarillento; márgenes lisos, lobados u ondulados, hacia la parte superior se contornean; talo de $2.6-7.3 \mathrm{~cm}$ de longitud, de 0.5-6 mm de ancho, cilíndrico de la base al centro, se va aplanando hacia el ápice; células sin un patrón de organi- zación en vista superficial, de formas variadas, rectangulares con bordes redondeados, cuadradas, ovaladas e irregulares, de 5-12.5 $\mu \mathrm{m}$ de ancho, de 7.5-17.5 $\mu \mathrm{m}$ de largo.

Ejemplares examinados: MÉXICO. Tabasco, Paraíso, Playa Paraíso Escollera Oeste, 15.VIII.1989, D. León GM391 (FCME); Cárdenas, Puerto Andrés Sánchez Magallanes, 2.IV.2011, J. Zamudio CA114 (UJAT); 27.X.2012, N. Quiroz et al. CA140 (UJAT); 02.IX.2012, O. Hernández CA246 (UJAT).

Referencias: Mendoza-González y Mateo-Cid, 1996; Littler y Littler, 2000; Dawes y Mathieson, 2008.

Ulva lactuca L. Figs. 4D; 5B.

Talo laminar de color verde claro con márgenes ondulados, base en forma de cuña; talo de 3.1-7-4 cm de largo, de 0.8-1.4 cm de ancho; células con disposición irregular en vista superficial, ovaladas, poliédricas a irregulares; en corte transversal se observa una separación entre membranas de $5 \mu \mathrm{m}$, células más largas que anchas de $5-10 \mu \mathrm{m}$ de ancho, de 15-20 $\mu \mathrm{m}$ de largo.

Ejemplares examinados: MÉXICO. Tabasco, Paraíso, Puerto de Dos Bocas, 26.V.2008, M. Ricardez CA89 (UJAT); Cárdenas, Puerto Andrés Sánchez Magallanes, 02.IV.2011, J. Zamudio CA211 (UJAT).

Referencias: Taylor, 1960; Littler y Littler, 2000; Ceballos, 2008.

Ulva prolifera O.F. Müller. Fig. 4F.

Talo filamentoso, tubular, color verde claro, crecimiento gregario como un mechón de pelo; márgenes de los filamentos lisos, con ligeras ondulaciones y constricciones; talo de 2.5-4.6 cm de largo, de 0.5-2 mm de diámetro; ramificación hacia la base, se observa un eje principal del que parten las ramas; la ramificación disminuye hacia la parte media y apical; células con una organización longitudinal y transversal en vista superficial, de forma rectangular a irregulares de 4-10.8 $\mu \mathrm{m}$ de largo, de 4.2-7.2 $\mu \mathrm{m}$ de diámetro. 


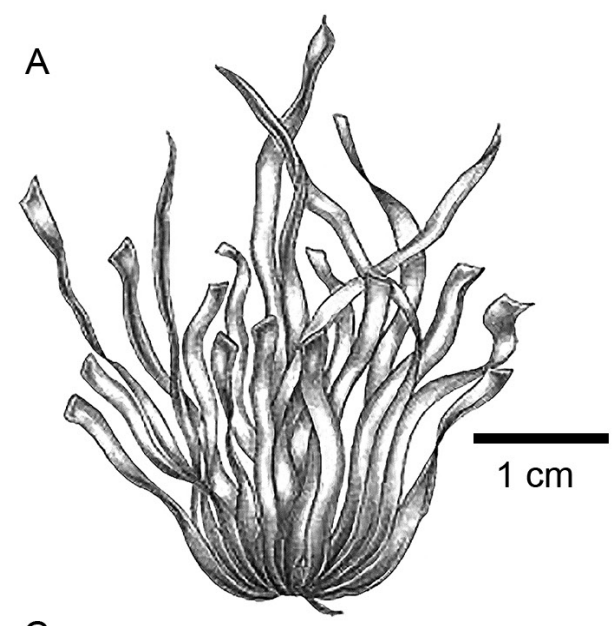

C
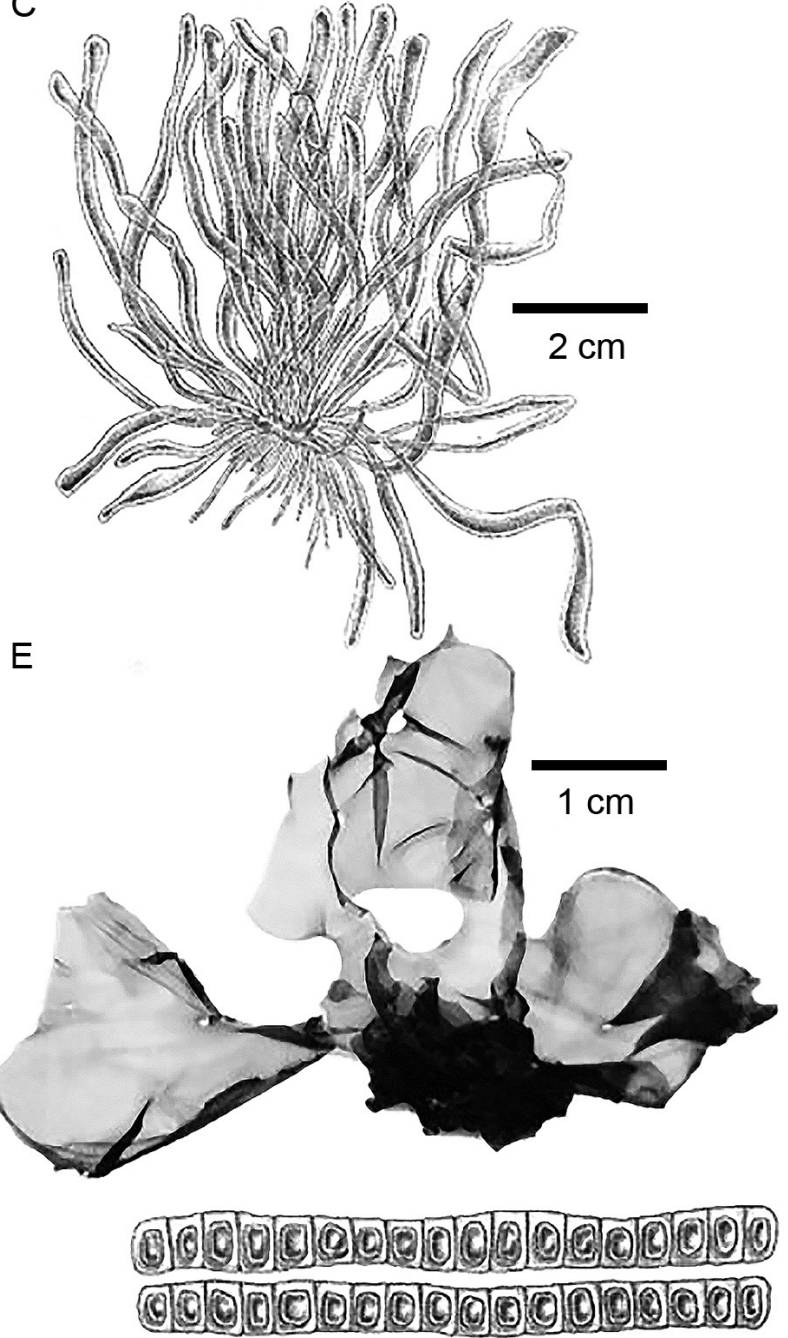

$40 \mu \mathrm{m}$

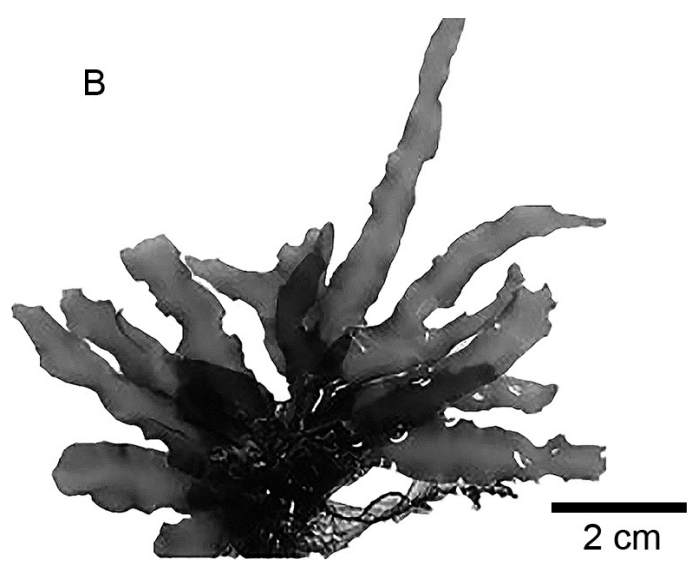

D

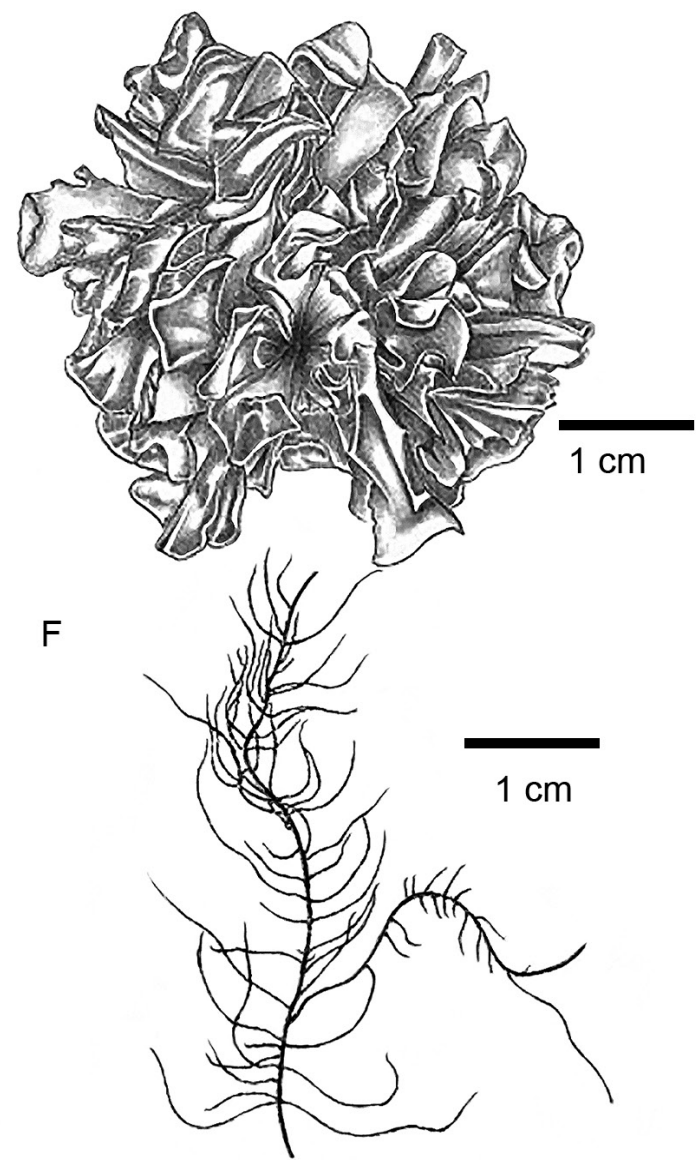

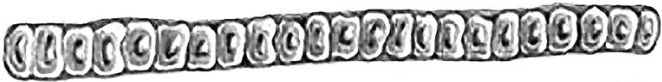
(190000000000000000

Figura 4: A. talo de Ulva compressa L.; B. talo de Ulva fasciata Delile., se observan láminas con forma de cinta; C. talo de Ulva intestinalis L.; D. talo de Ulva lactuca L., con frondas rizadas; E. talo de Ulva rigida C. Agardh.; F. talo de Ulva prolifera O.F.Müller., se observan las ramificaciones; G. corte transversal de $U$. compress $a$ L.; H. corte transversal de $U$. linza L. 
(A)

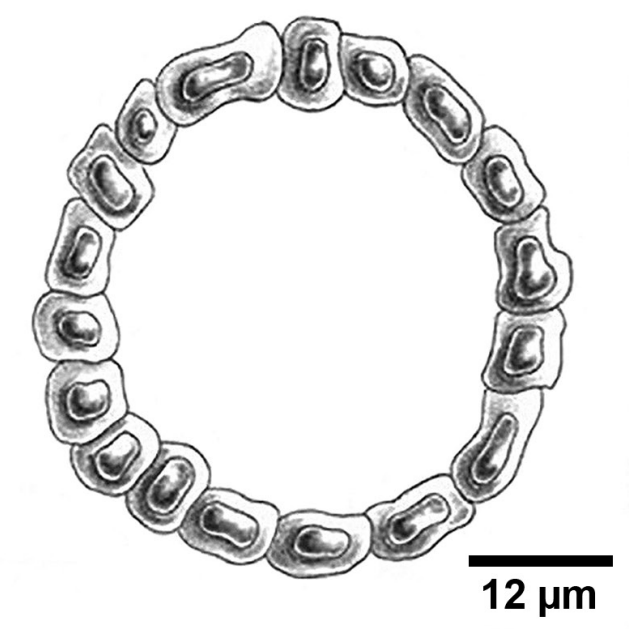

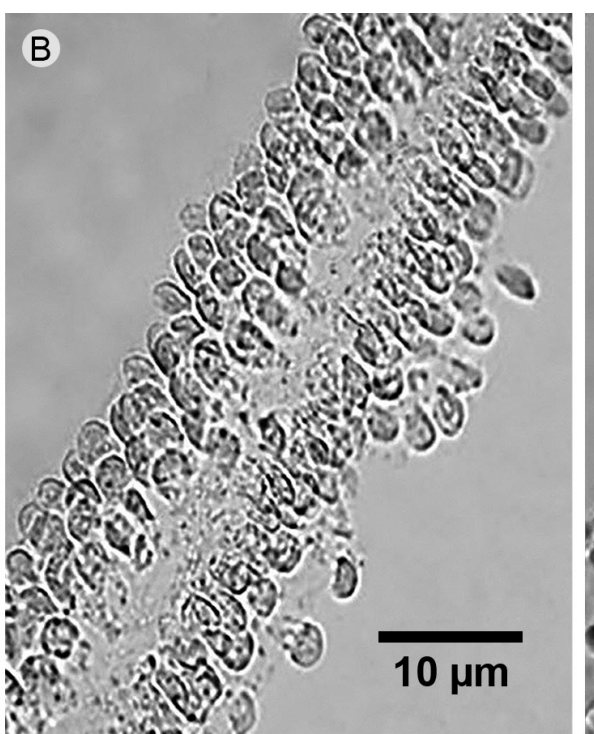
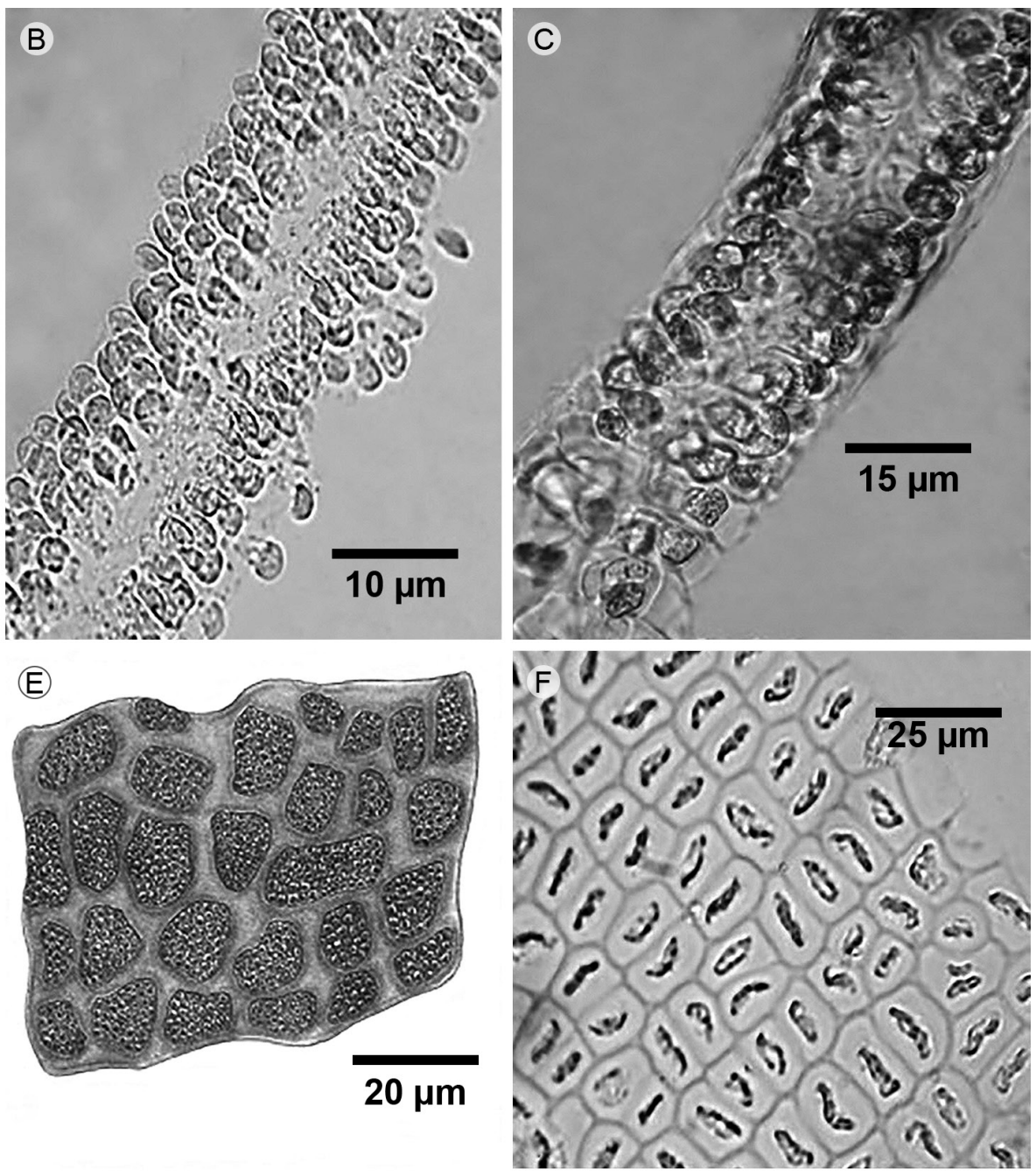
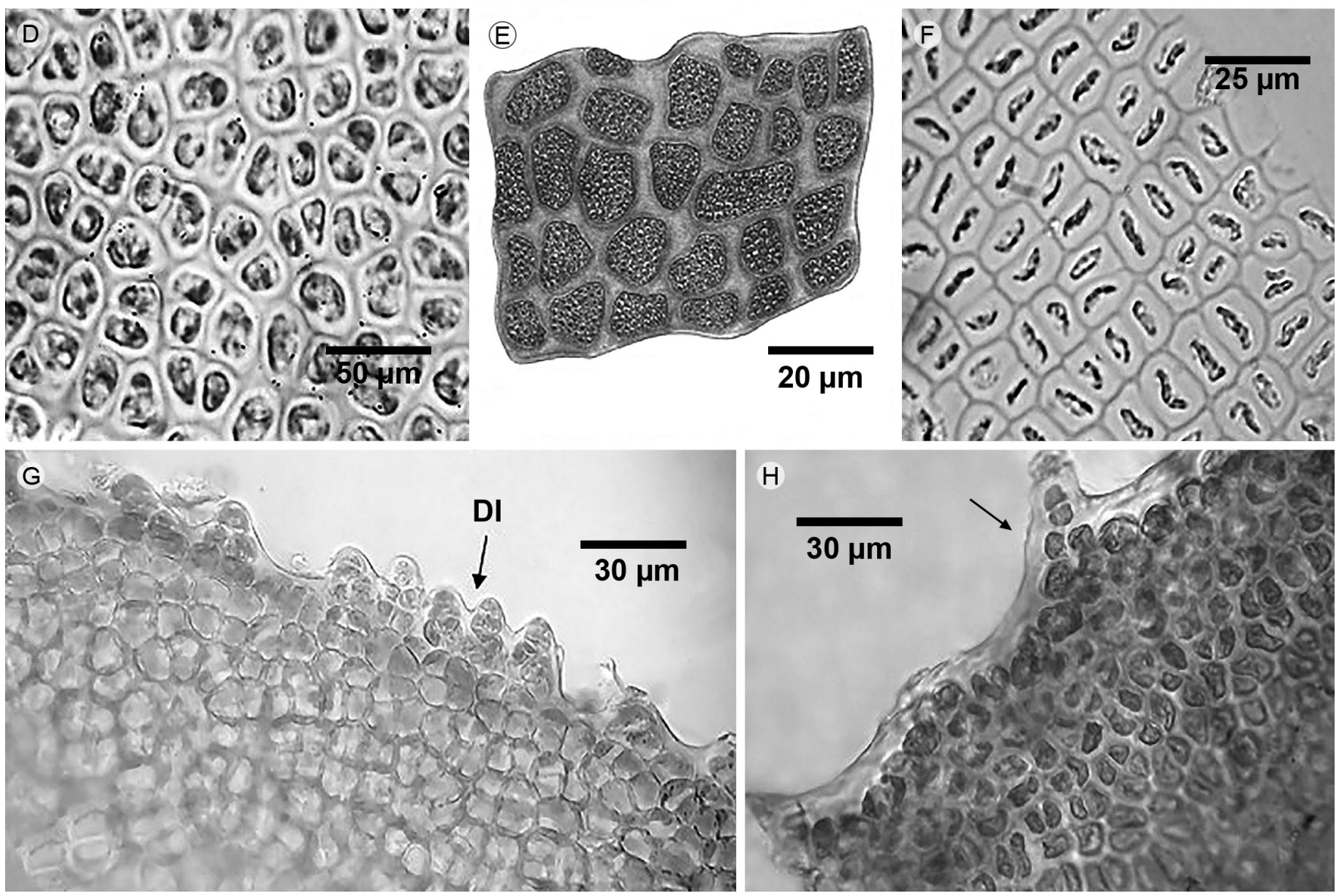

Figura 5. A. corte transversal de Ulva intestinalis L.; B. corte transversal de Ulva lactuca L., dos células de grosor; C. corte transversal de Ulva rigida C. Agardh.; D. vista superficial de las células de Ulva fasciata Delile., células con forma y disposición irregular; E. vista superficial de las células de Ulva intestinalis L., de forma irregular y sin un patrón de acomodo; F. vista superficial de las células de Ulva flexuosa; G. y H. talo de Ulva rigida, células con disposición irregular, dentículos intermitentes (DI). 
Ejemplares examinados: MÉXICO. Tabasco, Paraíso, Playa Paraíso Escollera Oeste, 15.VIII.1989, D. León CA2 (UJAT).

Referencia: Taylor, 1960; Hayden et al., 2003; Dawes y Mathieson, 2008.

Ulva rigida C. Agardh. Figs. 4E; 5C, $\mathrm{H}$.

Talo laminar de color verde oscuro de 3-14 cm de largo; láminas rizadas, de márgenes ondulados, abundantes dentículos microscópicos intermitentes; células en vista superficial sin un patrón de acomodo, redondeadas, cuadradas e irregularmente poliédricas, de 3-8 $\mu \mathrm{m}$ de diámetro; pirenoides 1 o 2 por célula.

Ejemplares examinados: MÉXICO. Tabasco, Cárdenas, Puerto Andrés Sánchez Magallanes, 27.X.2012, N. Quiroz et al. CA131 y CA132 (UJAT); 27.X.2012, I. Torres y C. Guerrero CA163 y CA203 (UJAT); 02.IX.2012, O. Hernández CA243 (UJAT).

Referencia: Littler y Littler, 2000.

\section{Discusión}

La temperatura media anual superficial del agua de las costas de Tabasco es de $25^{\circ} \mathrm{C}$, lo que indica que la ficoflora aquí listada queda comprendida dentro del intervalo de distribución tropical de algas marinas en relación con la temperatura del agua (Dawes, 1991). Lo mismo fue señalado por Sentíes y Dreckmann (2013), quienes mencionan que la ficoflora litoral del estado está cercana a iniciar una zona de transición hacia la flora caribeña, más que hacia la templada.

Las especies previamente reportadas por Sentíes y Dreckmann (2013) para Tabasco (Cuadro 4) no se encontraron durante el desarrollo de este trabajo, a pesar de que las épocas de muestreo en ambos estudios fueron las mismas, difiriendo únicamente un año entre éstos. Sin embargo, solo se comparte una localidad entre los trabajos, que es el Puerto Andrés Sánchez Magallanes. El resto de las localidades exploradas durante este estudio no fueron visitadas por Sentíes y Dreckmann (2013), lo que podría explicar la diferencia entre las especies reportadas en los dos trabajos. Respecto a las épocas de muestreo, el mayor número de especies se encontró en la temporada de lluvias, coincidiendo con lo reportado por otros autores para la región (Ortega et al., 2001; Callejas-Jiménez et al., 2005).

Según lo presentado por Ortega et al. (2001), Mateo-Cid et al. (2013) y Sentíes y Dreckmann (2013), las Ulvophyceae determinadas en el área de estudio presentan afinidad con las reportadas para Campeche y Veracruz. De las 17 especies descritas en este trabajo, 15 se han reportado para el litoral de Campeche, mientras que ocho forman parte de la flora algal de Veracruz, mostrando una distribución continua entre las tres entidades federativas. Especies como Ulva lactuca, U. fasciata, U. prolifera, $U$. flexuosa y Rhizoclonium riparium fueron reportados por Garduño-Solórzano et al. (2005) como de distribución continua entre los estados del Golfo de México.

La afinidad ficoflorística de Tabasco respecto a los estados adyacentes puede explicarse por la existencia de corrientes costeras provenientes de la plataforma de Tamaulipas, que circulan sobre Veracruz y Tabasco, hasta llegar a Campeche principalmente en otoño e invierno ( $\mathrm{Za}$ vala et al., 2005). Estas corrientes tropicales, aunadas a los intervalos de temperatura y características físicas de la costa como la presencia y tipo de sustrato, determinan cuales

Cuadro 4: Especies reportadas previamente por Sentíes y Dreckmann (2013) para las costas de Tabasco.

\section{ULVOPHYCEAE}

Anadyomenaceae

Anadyomene menziesii (J.E. Gray) J. Agardh

Caulerpaceae

Caulerpa microphysa (Weber-van Bosse) Feldmann

C. peltata J.V. Lamour.

C. prolifera (Forssk.) J.V. Lamour.

Codiaceae

Codium isthmocladum Vickers 
especies migran y cuales se establecen (Ramírez, 1995).

Tomando en cuenta los resultados de este estudio y los registros previos de otros autores, el número de especies de algas marinas para Tabasco asciende de 50 a 67, lo que representa $6.3 \%$ del número total de macroalgas marinas citado para el Atlántico mexicano (Pedroche y Sentíes 2003), así como $11 \%$ del número de especies para el Golfo de México (Ortega et al., 2001). Con relación al número de Ulvophyceae registradas para el Golfo de México, este trabajo presenta $14 \%$ de las especies dadas a conocer para esta región.

En comparación con el resto de los estados costeros del país, Tabasco presenta una riqueza específica baja de Chlorophyta, sobre todo si se toma en cuenta que el resto de las entidades, excepto Chiapas y Colima, tienen valores por encima de las 30 especies en sus inventarios florísticos (Pedroche et al., 2009). En relación a los estados del Atlántico mexicano, la diferencia en el número de especies de Tabasco con Tamaulipas, Campeche, y especialmente con Quintana Roo, Veracruz y Yucatán, son considerables. Sin embargo, estos sitios han sido ampliamente explorados, mientras que en Tabasco únicamente se han trabajado 12 localidades hasta el momento.

Se observa la mayor riqueza específica en las localidades con escolleras (Playa Paraíso y Puerto Andrés Sánchez Magallanes), lo cual coincide con lo indicado por Mendoza-González y Mateo-Cid, (1996). Según Taylor (1960) las estructuras de fijación tales como discos o hapterios se adhieren mucho mejor a las rocas que a cualquier otro sustrato, posibilitando así el incremento de su diversidad, mismas que fueron las formas de adhesión al sustrato más frecuentes en los ejemplares descritos.

Taylor (1960) señala que los sustratos expuestos al oleaje, como las escolleras, exhiben gran diversidad de algas marinas. En el Puerto Andrés Sánchez Magallanes se encontraron especies que toleran la exposición al fuerte oleaje y a la insolación, es el caso de Chaetomorpha antennina, Ulva fasciata y U. rigida. En la escollera Oeste de Playa Paraíso, las algas están sujetas a condiciones similares que las del Puerto Andrés Sánchez Magallanes; allí se encontraron Caulerpa sertularioides f. brevipes, Chaetomorpha antennina, U. fasciata, U. flexuosa y $U$. intestinalis.

La baja riqueza especifica en Centla puede atribuirse a la falta de sustratos que favorecen el desarrollo de macroalgas, ya que dicho sitio se caracteriza por la presencia de playas limo-arcillosas, y las desembocaduras de los ríos González, Grijalva, y San Pedro y San Pablo, que modifican el aporte de nutrientes, sedimentos y salinidad de la costa. Respecto a los sustratos, $46 \%$ de las especies se encontraron en los de tipo artificial, predominando las rocas formadoras de escollera. Esta influencia de origen humano es cada vez más común; los sustratos artificiales comenzaron a citarse en estudios ficológicos a partir de 1958 (Garduño-Solórzano et al., 2005). Estos autores encontraron a Chaetomorpha antennina y a Ulva fasciata desarrollándose en escolleras, a U. flexuosa y U. compressa L. creciendo sobre madera, sustrato en él que también crece $U$. intestinalis. En cuerdas de lancha reportan a Rhizoclonium riparium, mientras que Ulothrix flacca fue observada en cables de polipropileno. Estos últimos no se registraron durante el desarrollo de este estudio, donde las algas se encontraron creciendo en bolsas de plástico, costales o telas.

Las conchas del ostión (Crassostrea virginica) fueron el sustrato de origen animal más común. En general los organismos con concha son considerados hospederos de numerosas comunidades algales, ya que representan una excelente forma de dispersión tal como señala Ramírez (1995). Para el caso de este estudio se encontraron algas sobre algunos caracoles con vida que no fueron identificados taxonómicamente. Once \% de las algas estaban flotando, especies como Rhizoclonium riparium, Chaetomorpha gracilis y Ulotrhix flacca también fueron reportadas como flotadoras por Garduño-Solórzano et al. (2005).

El género Ulva presentó la mayor riqueza específica (7 especies) así como la mayor distribución en el estado de Tabasco, encontrándose en cuatro de las siete localidades consideradas en este trabajo. Esto se relaciona con la capacidad que tienen las especies de este género para crecer en distintos hábitats con grandes diferencias de 
salinidad, temperatura, turbidez y composición química, como son los ambientes muy enriquecidos con nutrientes procedentes de los ríos o en zonas donde existen contaminantes. Además, se fijan a diferentes tipos de sustratos duros, como rocas, maderas y cuerdas entre otros (Rast y Holland, 1988; Cano-Mallo, 2008). Esta alta capacidad para tolerar condiciones que para otras algas pueden tornarse adversas les permiten a las especies de Ulva prosperar en ambientes como los de Tabasco.

Chaetomorpha nodosa ha sido registrada en Brasil, Chile, Guyana y Venezuela (Taylor, 1960; Wynne, 2011; Guiry y Guiry, 2016), siendo la primera vez que se reporta para las costas de México. Es posible que algas como Cladophora sp. requieran más adelante el desarrollo de estudios moleculares, ya que sus características morfológicas no permiten una clara identificación a nivel de especie.

\section{CONCLUSIONES}

Se considera que a pesar del aporte que representa este trabajo al conocimiento de la ficoflora de Tabasco, es indispensable seguir realizando exploraciones ficológicas a lo largo de la costa y actualizar la información acerca de las especies de la región. Datos como su distribución, estacionalidad, características morfológicas, anatómicas y reproductivas son necesarias y pueden encaminar al desarrollo de futuras investigaciones aplicadas para el aprovechamiento de estos recursos.

\section{CONTRIBUCIONES DE AUTORES}

NQ, DL Y MR llevaron a cabo las recolectas de material en las áreas de muestreo. NQ realizó la revisión del material de herbario, la elaboración de preparaciones, la toma de medidas, así como la identificación y descripciones de los ejemplares. DL y MR corroboraron la identificación taxonómica. NQ escribió el manuscrito con el apoyo de DL y MR. Todos los autores contribuyeron a la discusión, revisión y aprobación del manuscrito final.

\section{FINANCIAMIENTO}

El estudio no tuvo financiamiento por parte de ningún proyecto.

\section{AgradeCIMIENTOS}

Agradecemos a Silvia Capello García y María de los Ángeles Guadarrama por el apoyo brindado con los equipos e instalaciones para el procesamiento e identificación de las muestras, a Miguel A. Ortiz Ramos y Diego A. Falcón Vidal por su apoyo en campo. A Carolina Campos por su apoyo con las ilustraciones.

\section{LITERATURA CITADA}

Ardito, S. y M. García. 2009. Estudio ficológico de las localidades de Puerto Francés y San Francisquito, Estado Miranda, Venezuela. Acta Botanica Venezuelica 32(1): 113143.

Callejas-Jiménez, M. E., A. Sentíes y K. M. Dreckmann. 2005. Macroalgas de Puerto Real, Faro Santa Rosalía y Playa Preciosa, Campeche, México, con algunas consideraciones florísticas y ecológicas para el estado. Hidrobiológica 15(1): 89-96.

Cano-Mallo, M. 2008. Bases biológicas de Ulva fasciata Delile, (Chlorophyta) para su posible explotación, al oeste de la Habana, Cuba. Tesis de doctorado. Universidad de la Habana. La Habana, Cuba. 150 pp.

Castellaro, G., F. Squella, T. Ullrich, F. León y A. Raggi. 2007. Algunas técnicas microhistológicas utilizadas en la determinación de la composición botánica de dietas de herbívoros. Agricultura Técnica 67(1): 86-93. DOI: http:// dx.doi.org/10.4067/S0365-28072007000100011

Ceballos, B. 2008. Las Clorofitas marinas (Chlorophyta) en el arrecife Ingeniero (Penacho del Indio), Mpio. de Boca del Rio, Veracruz. Tesis de licenciatura. Universidad Veracruzana. Veracruz, México. 89 pp.

Dawes, C. 1991. Botánica marina. Ed. Limusa. México, D.F., México. 673 pp.

Dawes, C. y A. Mathieson. 2008. The seaweeds of Florida. University Press of Florida. Gainesville, USA. 592 pp.

Dreckmann, K. y G. De Lara-Isassi, 2000. Gracilaria caudata J. Agardh (Gracilariaceae, Rhodophyta) en el Atlántico mexicano. Hidrobiológica 10(2): 125-130.

Dreckmann, K., A. Sentíes, F. Pedroche y M. Callejas 2006. Diagnóstico florístico de la ficología marina bentónica en Chiapas. Hidrobiológica 16(2): 147-158. 
Garduño-Solórzano, G., J. Godínez-Ortega y M. Ortega. 2005. Distribución geográfica y afinidad por el sustrato de las algas verdes de las costas del Golfo de México y Mar Caribe. Boletín de la Sociedad Botánica de México 76: 61-78.

González-González, J., M. Gold-Morgan, H. León-Tejeda, C. Candelaria, D. León-Álvarez, E. S. Zaragoza y D. Fragoso. 1996. Catálogo onomástico (nomenclator) y bibliografía indexada de las algas bentónicas marinas de México. Cuadernos No. 34. Instituto de Biología, Universidad Nacional Autónoma de México. México, D.F., México. 492 pp.

Guiry, M. y G. Guiry. 2016. AlgaeBase. World-wide electronic publication, National University of Ireland, Galway. http:// www.algaebase.org; Consultado el 25 de mayo de 2015.

Hayden, H., J. Blomster, C. Maggs, P. Silva, M. Stanhope y J. Waaland. 2003. Linnaeus was right all along: Ulva and Enteromorpha are not distinct genera. European Journal of Phycology 38(3): 277-294. DOI: http://dx.doi. org/10.1080/1364253031000136321

Littler, D., M. Littler, K. Bucher y J. Norris. 1989. Marine plants of the Caribbean, a field guide from Florida to Brazil. Smithsonian Institution Press. Washington, D.C., USA. 263 pp.

Littler, D. S. y M. M. Littler. 2000. Caribbean reef plants: an identification guide to the reef plants of the Caribbean, Bahamas, Florida and Gulf of Mexico. Offshore Graphics. Washington, D.C., USA. 542 pp.

Lot, A. y F. Chiang. 1986. Manual de herbario. Consejo Nacional de la Flora de México, A.C., México, D.F., México. 142pp.

Mateo-Cid, L., C. Mendoza-González, A. Ávila-Ortiz y S. Diaz-Martinez. 2013. Algas marinas bentónicas del litoral de Campeche, México. Acta Botanica Mexicana. 104: 53-92.

Mendoza-González, C. y L. Mateo-Cid. 1996. Contribución al estudio de la ficoflora marina de la costa del estado de Chiapas, México. Polibotánica 2: 61-118.

Miranda, A., C. Wallace, G. Limoeiro y L. Souza. 2009. Os gêneros Chaetomorpha Kütz. nom. cons. e Rhizoclonium Kütz. (Chlorophyta) do litoral do Estado da Bahía, Brasil. Revista Brasileira Botanica 32(3): 545-570. DOI: http:// dx.doi.org/10.1590/S0100-84042009000300014
Morón, M. y S. Ardito. 2010. Estudio ficológico del litoral rocoso de Isla Larga, Parque Nacional San Esteban, estado Carabobo, Venezuela. Acta Botanica Venezuelica 33(2): 167-185.

Orozco, H. y K. Dreckmann. 1995. Macroalgas estuarinas del litoral del Golfo de México. Cryptogamie Algologie 16(3): 189-198.

Ortega, M. M., J. L. Godínez y G. Garduño Solórzano. 2001. Catálogo de algas bénticas de las costas mexicanas del Golfo de México y Mar Caribe. Comisión Nacional para el Estudio de la Biodiversidad y Universidad Nacional Autónoma de México. México, D.F., México. 594 pp.

Pedroche, F. y A. Sentíes. 2003. Ficología marina mexicana. Diversidad y Problemática actual. Hidrobiológica 13(1): 23-32.

Pedroche, F., A. Sentíes, E. Novelo y M. Meave. 2009. Tomo II: Ciencias biológicas: algas, pasado, presente y futuro en México. In: Ramírez, J. (ed.). Cosmos: Enciclopedia de las ciencias y la tecnología en México. Universidad Autónoma Metropolitana-Iztapalapa; Consejo Nacional de Ciencia y Tecnología. México, D.F., México. 55-69 pp.

Peruzzi, V., D. Rezende, N. Marcello, Y. Yoneshigue y R. Clapis. 2009. Four new additions to the marine flora of Fernando de Noronha Archipelago, Tropical western South Atlantic Ocean. Check List 5(2): 210-215.

Ramírez, A. 1995. Estudio preliminar de las algas rojas (Rhodophyta) del litoral del Estado de Tabasco. Tesis de licenciatura. Universidad Nacional Autónoma de México. Tlalnepantla, Estado de México, México. 66 pp.

Rast, W. y M. Holland. 1988. Eutrophication of lakes and reservoirs, a framework for making management decisions. AMBIO 17(1): 2-12.

Sánchez, A. y E. Barba. 2005. Capítulo I: Biodiversidad de Tabasco. In: Bueno, J., F. Álvarez y S. Santiago (ed.). Biodiversidad del Estado de Tabasco. Instituto de Biología. Universidad Nacional Autónoma de México-Comisión Nacional para el Conocimiento y Uso de Biodiversidad. México, D.F., México. 1-16 pp.

Sentíes, A. y K. Dreckmann. 2013. Lista actualizada de las macroalgas de Tabasco, México. Acta Botanica Venezuelica 36(2): 109-117. 
Solé, M. y P. Pardo. 2010. Ficoflora Marina del Sur de la Península de Macanao, Isla de Margarita, Venezuela. II. Rhodophyta. Acta Botanica Venezuelica 33(2): 187-211.

Taylor, W. R. 1960. Marine algae of the eastern tropical and subtropical coast of the Americas. University of Michigan Press. Ann Arbor, USA. 870 pp.

West, R., N. Psuty y B. Thom. 1985. Las tierras bajas de Tabasco en el Sureste de México. Instituto de Cultura de Tabasco. Villahermosa, México. 416 pp.

Wynne, M. J. 2011. A checklist of benthic marine algae of the tropical and subtropical western Atlantic: third revision. Nova Hedwigia Beiheft. 140: 1-166.
Zavala, J., O. Salmerón, V. Aguilar, S. Cerdeira y M. Kolb. 2005. Caracterización y regionalización de los procesos oceanográficos de los mares mexicanos. Comisión Nacional para el Conocimiento y Uso de la Biodiversidad (CONABIO) http://www.conabio.gob.mx/gap/index.php/ Procesos_oceanogr\%C3\%A1ficos (consultado en marzo de 2015). 